\title{
A Study on the Analysis Method of Passenger Flow in Airport Using Laser Sensor
}

\author{
Seok-Hee Lee ${ }^{1}$, Hyung-Taek Lee ${ }^{2}$, Nhu-Quynh Phan ${ }^{3}$ and Gwang-Yong Gim ${ }^{4 *}$ \\ ${ }^{1} 04143$ Solution \& Service Business Division, LG Hitachi., LG Mapo Bldg 155, \\ Mapo-daero, Seoul, Republic of Korea \\ ${ }^{2} 08380$ \#301, Ace Techno Tower 5, 20, Digital-ro 31-gil, Guro-gu, \\ Seoul, Republic of Korea \\ ${ }^{3} 06978$ Dept. Business Administration, Graduate School Soongsil Univ., \\ 369 Sangdo-ro, Dongjak-gu, Seoul, Republic of Korea \\ ${ }^{4} 06978$ Dept. Business Administration, Soongsil Univ., 369 Sangdo-ro, \\ Dongjak-gu, Seoul, Republic of Korea \\ 1'chris@lghitachi.cokr, ${ }^{2}$ htlee@innotium.com, ${ }^{3}$ phannhuquynh@hotmail.com, \\ 4*gygim@ssu.ac.kr
}

\begin{abstract}
There are wide and recent research efforts to extract location information of people in certain spaces, with new services using this information in real space being launched in the market.

In particular, there are emerging services and solutions that measure movement flow of people in airports, public facilities, commercial facilities, factories, warehouses, and other locations to mitigate congestion or enhance security and safety in these spaces.

Methods of measuring the movement flow of people include camera image analysis, Wi-Fi, beacon, and RFID, but these methods are inconvenient as the tracking targets must carry a certain medium (smartphone, RFID tag, etc.), while camera images infringe upon personal privacy, posing many problems for commercialization of these movement flow technologies.

In order to solve this problem, the current study applies a movement flow measurement technology using laser sensors, applying it to a Korean airport to verify its feasibility through an experiment.

The researchers propose a method to improve customer service by analyzing the passenger movement flow, congestion levels, and wait time at the airport using the acquired data.
\end{abstract}

Keywords: Flow Tracking Analysis, Big Data, Laser Sensor, Visualization, Congestion

\section{Introduction}

\subsection{Research Background}

As the importance of key technologies including artificial intelligence (AI), fintech, big data, and internet of things (IoT) is increasing with the advent of the Fourth Industrial Revolution and the prevalence of IoT rises, the use of vast amount of data collected and accumulated via cable and wireless networks is increasing in tandem. In the field of IoT, sensors are increasingly embedded in various devices, effectively establishing the infrastructure of sensor networks that collect a diverse range of data. In more cases, companies and organizations are measuring the

Received (December 17, 2017), Review Result (March 10, 2018), Accepted (March 19, 2018)

* Corresponding Author 
dynamic movement of people, turning them into big data and visualizing them to make facility and service operation more efficient. Airports, metro stations, and department stores are quantitatively measuring their customers' movement flow to reduce congestion and advertise products, and many services and solutions are enabling users to enhance security management $[11,12,13]$. Such solutions automatically recognize the images of people and objects to survey their flow of movement. While such method of using camera footages facilitates the application of various image processing technologies and reduces investment by utilizing preexisting camera equipment, it is limited in that sensor precision is affected by the strength of lighting or weather conditions and that the camera images can violate privacy. Some measurement systems require visitors to log-in using Wi-Fi or beacon, but their data collection volume can be inadequate, as the targeted individuals must have a smartphone to access the data collection platform [1,2].

\subsection{Purpose of the Study}

The goal of the current study is to resolve such problems by applying laser sensors to people flow analysis. The laser sensor method employs a measurement technology using Class-1 laser systems, which does not require the tracking targets to own a mobile phone or a separate sensor while enhancing the stability and precision of the measurement. It also eliminates the concern about privacy as the system does not specify certain individuals. The method can be used to analyze the flow of people and objects to acquire information about the people size, flow of targets, and length of stay, in addition to analyzing and utilizing such location information in connection with BI tools and AI systems. The current study is the first empirical experiment in Korea to apply the laser sensor measurement technology in an airport to understand the feasibility of the proposed system, which analyzes the travel flow, congestion, and wait times [14, 15, 16, 18]. The objective is to suggest techniques to visualize the collected flow information and enhance customer service.

\section{Research}

\subsection{Overview of Movement Flow Analysis}

Movement flow analysis solution refers to a system that uses laser sensors, wireless LAN platforms, RFID tags, and beacons to measure, collect, and analyze the location and flow of individuals within a certain space, in order to use the information for customer marketing and service improvement $[6,7,8]$. As displayed in Figure 1, The laser is projected onto the target, with laser sensors picking up the reflected light to measure the distance. For example, laser sensors are used to detect the targets (people or objects) real-time with high precision to visualize their movement; a constant tracking scheme measures the location, movement (direction and speed), and trajectory of the targets, with the results accumulated on to a control $\mathrm{PC}$ as a coordinate data. The data is then analyzed later to create various reports and visualized products. Trajectory display, heat map, and people count were the visualization methods used. 


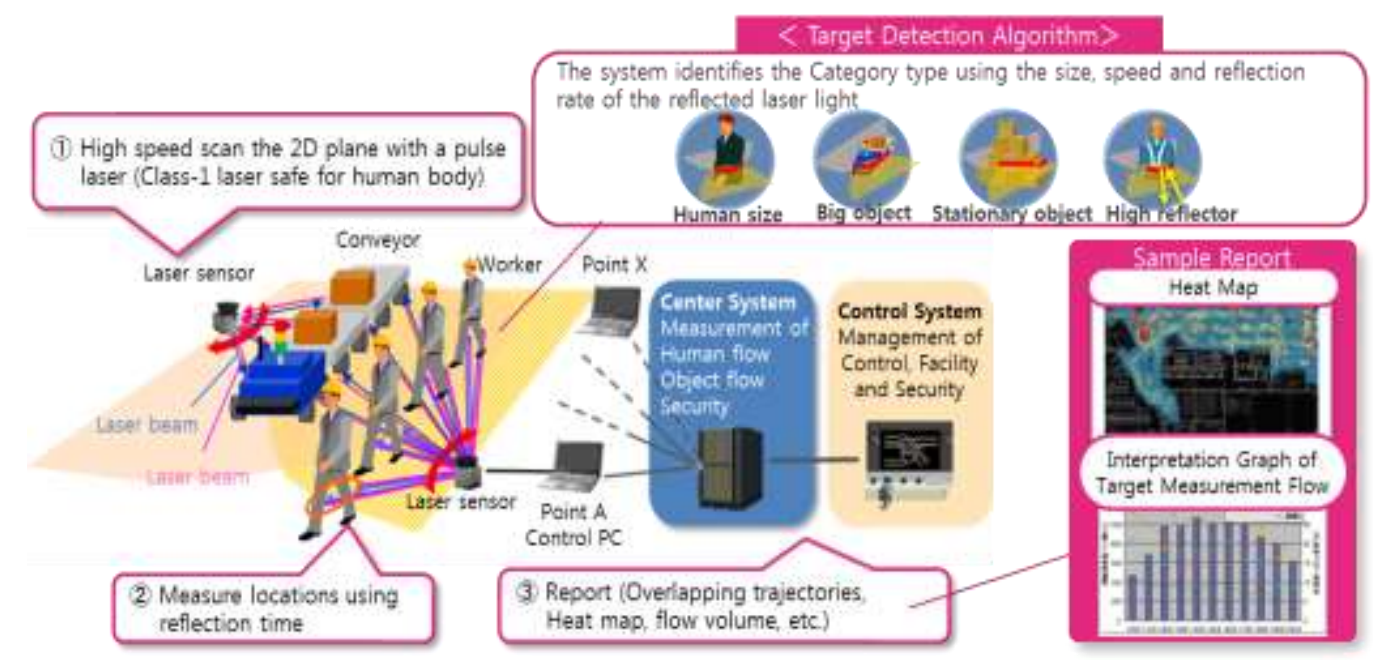

Figure 1. The Concept of the Movement Flow Analysis Solution

Location measurement technologies are critical in order to execute movement flow analysis. While the Global Positioning System (GPS) is the prevalent method for outdoor measurements, infrared (IR), radar, wireless lan (WLAN), RFID, and Bluetooth can be used for indoor measurements depending on the required measurement precision and distance. Table 1 summarizes the strengths, weaknesses, and features of various technologies used to analyze movement flow $[3,4,9,10]$.

Table 1. Comparison of Movement Flow Analysis Features

\begin{tabular}{|l|c|l|l|l|c|c|}
\hline Category & $\begin{array}{l}\text { Wireless } \\
\text { LAN }\end{array}$ & Zigbee & RFID & Beacon & CCTV & $\begin{array}{l}\text { Laser } \\
\text { Sensor }\end{array}$ \\
\hline $\begin{array}{l}\text { Recognition } \\
\text { Distance }\end{array}$ & Long & Short & Short & Long & Short & Long \\
\hline $\begin{array}{l}\text { Privacy } \\
\text { Concerns }\end{array}$ & Yes & Yes & Yes & Yes & Yes & No \\
\hline $\begin{array}{l}\text { Distance } \\
\text { Accuracy }\end{array}$ & Low & Medium & Medium & Low & Low & High \\
\hline $\begin{array}{l}\text { Target } \\
\text { Requires } \\
\text { Measurement } \\
\text { Device }\end{array}$ & Yes & Yes & Yes & Yes & Yes & No \\
\hline $\begin{array}{l}\text { Portability and } \\
\text { Ease of } \\
\text { Installation }\end{array}$ & $\begin{array}{c}\text { None } \\
\text { (Fixed) }\end{array}$ & Yes & Yes & $\begin{array}{c}\text { None } \\
\text { (Fixed) }\end{array}$ & $\begin{array}{c}\text { None } \\
\text { (Fixed) }\end{array}$ & Yes \\
\hline $\begin{array}{l}\text { Installation } \\
\text { Cost }\end{array}$ & Low & Medium & Medium & Low & High & Medium \\
\hline $\begin{array}{l}\text { Required } \\
\text { Measurement } \\
\text { Device for } \\
\text { Targets }\end{array}$ & Smartphone & Device & Tag & Smartphone & None & None \\
\hline $\begin{array}{l}\text { Use in the } \\
\text { Dark }\end{array}$ & Yes & Yes & Yes & Yes & None & Yes \\
\hline
\end{tabular}




\subsection{Laser Sensor Selection}

Model L-T1103-MRS57B by SICK Ltd., a German company was used in the measurement experiment [5, 17]. The laser sensor projects light onto each individual target and uses the time it takes for the light to be reflected back to the sensor to calculate the precise location and distance of the target. The sensor has a range of $270^{\circ}$, rotating the width of $0.3^{\circ}$ intervals while scanning with a frequency of $5 \mathrm{~Hz}$ and taking fifteen measurements per second. This enables it to accurately detect the location of the target before and after movement, allowing for calculation of the target's direction and amount of movement, as well as the trajectory.

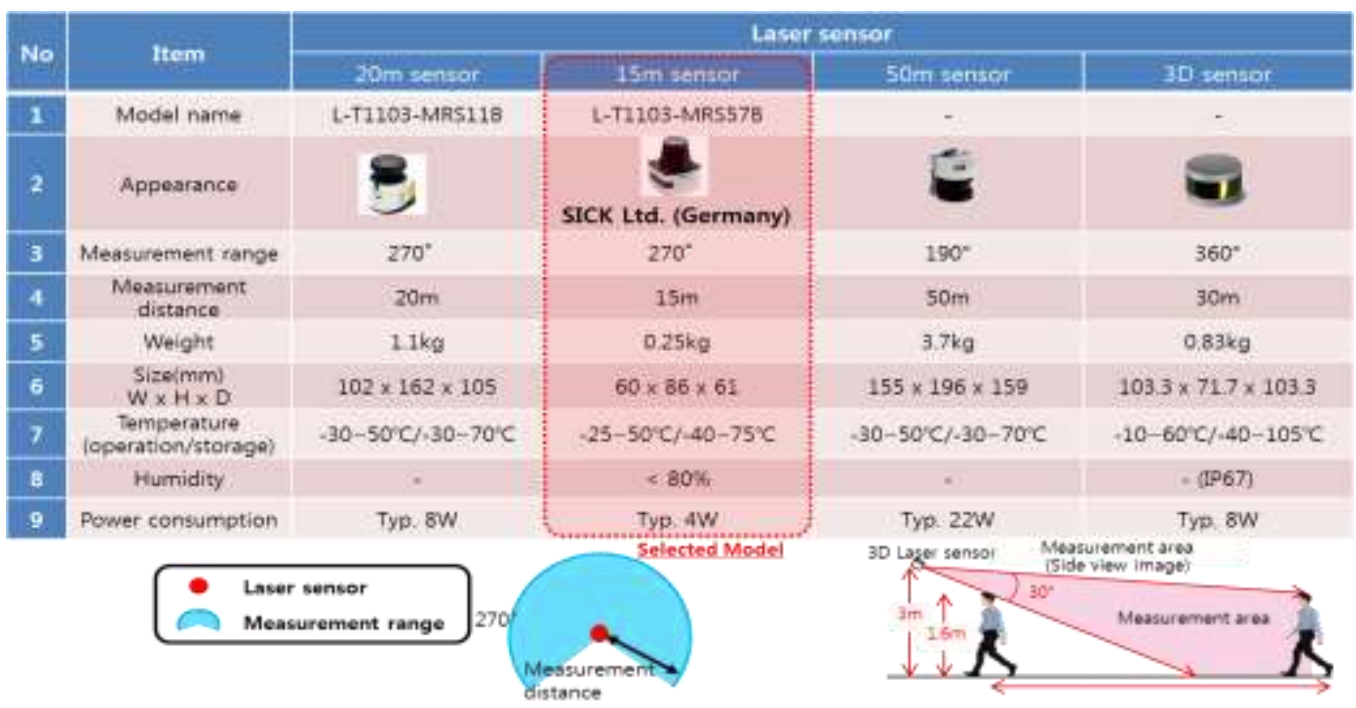

Figure 2. Types of Laser Sensors

Table 2 shows the location information delivered to the measurement PC or server from the laser sensor. Using only five data types, the system can identify the location, route, speed, and direction of the target, in addition to interpreting the data to display the trajectory of the target, time spent in passing through a certain point, as well as congestion levels and wait times in certain areas. These data can quantify the overall flow of movement for the user to identify problems and plan solutions. In particular, the system provides vector values and therefore can display the movement trajectory of the targets. In addition, it can calculate the targets' speed to identify whether people are stopped, walking, or running, providing more useful resources. As the current study used 2D laser sensor, among the $\mathrm{X}, \mathrm{Y}$, and $\mathrm{Z}$ axes in the location information, the $\mathrm{Z}$ value is expressed as 0 (Zero) on all data points.

Table 2. Information Parameters from the Laser Sensor Measurement

\begin{tabular}{|l|l|l|}
\hline No. & Parameter & Details \\
\hline $\mathbf{1}$ & Trajectory ID & $\begin{array}{l}\text { ID randomly assigned to the system for each object; } \\
\text { the same objectives are consistently marked with the same IDs }\end{array}$ \\
\hline $\mathbf{2}$ & Time & $\begin{array}{l}\text { In 200ms intervals, the time printed for trajectory ID } \\
\text { information }\end{array}$ \\
\hline $\mathbf{3}$ & $\begin{array}{l}\text { Location Information } \\
\text { (X, Y, and Z axes) }\end{array}$ & $\begin{array}{l}\text { Location of the trajectory ID at certain times } \\
\text { (X, Y, and Z axes) }\end{array}$ \\
\hline $\mathbf{4}$ & Speed & Speed of the trajectory ID at certain times, expressed in $\mathrm{m} / \mathrm{s}$ \\
\hline $\mathbf{5}$ & Vector & Vector of the trajectory ID at certain times (direction) \\
\hline
\end{tabular}




\subsection{System Configuration}

As shown in Figure 3, the system consists of three modules, with each executing the following functions.

The measurement/management PC receives from the sensors the movement of the target at each second. This operation is executed real-time, and depending on the network condition of the measurement location, the data can be sent using TCP/IP or wireless LAN. Each measurement PC can access up to six laser sensors. The database PC stores the information received from measurement/management PC in the database. The interpretation PC uses a big data solution to analyze and visualize the collected movement flow data.

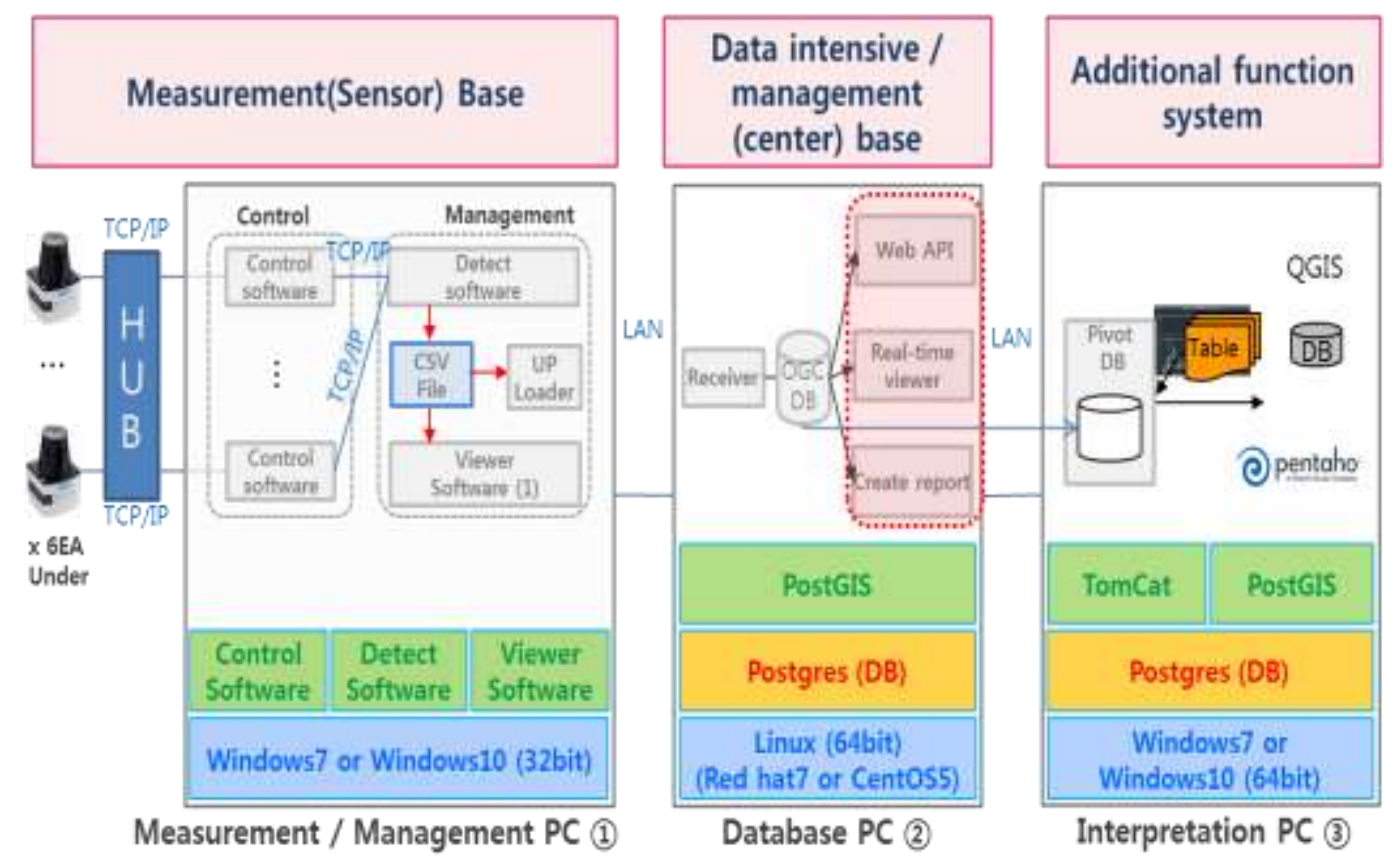

Figure 3. Configuration of the Measurement System (H/W \& S/W)

\subsection{Laser Sensor Installation}

One sensor is installed in the carouse \#l to detect the queue in front of the carousel \#1 from its interior, and another one is installed to count entrances through the right-hand side gate and queue on the right-hand side. Four more sensors are located to count entrances through the right-hand side gate and lack-side queue; movement flow near the right-hand side gate; back-side queue; movement flow near the exit and back-side queue. A simulator was used to confirm that there are no blind spots. The six laser sensors were evenly installed in the space from the arrival gate and carousel \#1 as shown in Figure 4, and the surveyed was partitioned into $1 \mathrm{~m} \times 1 \mathrm{~m}$ sections for the system before beginning the measurement of passenger movement flow. Instead of fixing them on the ceiling as in the case of CCTVs, the laser sensors can be conveniently clipped onto a tripod, stick, or other structures. This makes the installation and sensor location changes easy. After installing the sensors, a simulator was used to view the laser lights radiating from the devices, painted red in Figure 4, to check if detection areas overlap. In case the location of the sensors was changed, they were calibrated to ensure that the measurement was executed under optimal conditions. 


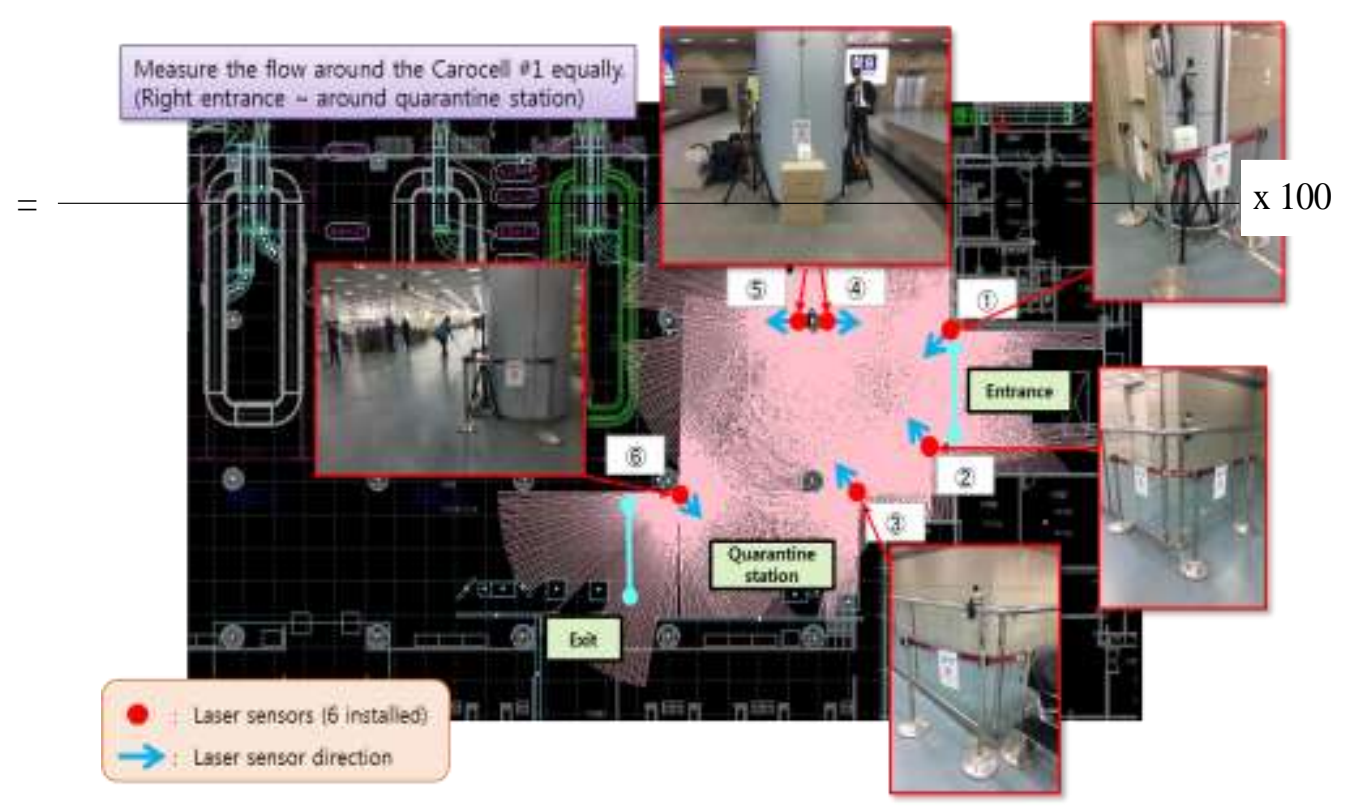

Figure 4. Installation of Laser Sensors

\subsection{Measurement Range}

Figure 5 shows the measurement area and count lines set for the current study. The yellow dotted line around the carousel \#1 indicates the analysis area in which the passengers wait to claim their baggage. A line was established at the right-hand side gate to count the number of passengers going through it to claim their baggage after the immigration interview. In addition, another count line was set at the exit gate to detect the number of passengers going out to the arrivals gate after claiming their baggage at carousel \#1.

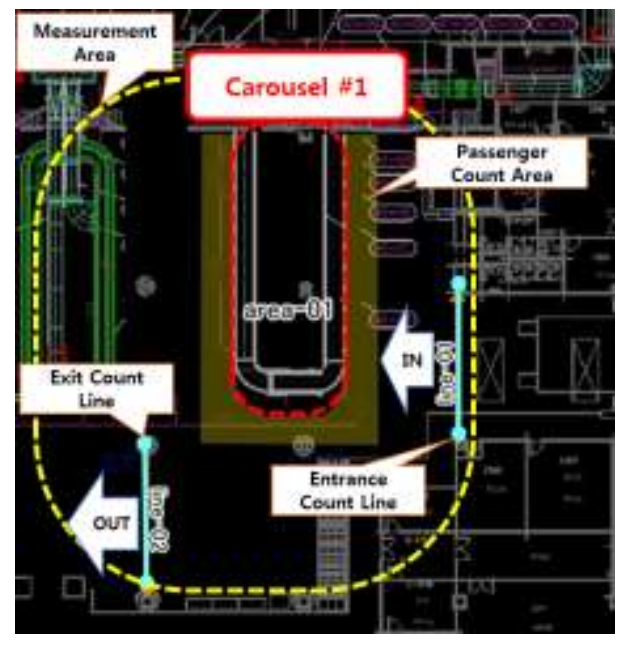

Figure 5. Range of the Measurement System

Each measurement area was partition into a grid of $1 \mathrm{~m} \times 1 \mathrm{~m}$, and the time that each target spent waiting in the area was calculated. The researchers divided a number of all grids by the number of grids that had people spending more than thirty seconds in one minute, in order to calculate the area occupation rate.

That is, Area Occupation Rate

Number of grids where people stayed for more than 30 seconds in 1 minute 
Number of all grids in the passenger measurement area

The count of entering people is the number of targets passing through Line-01 from right to left, and the count of exiting people is the same passing through Line-02 from left to right.

\subsection{Results of the Count}

The researchers compared the number of arriving passengers received from the airport and the line count data from the laser sensors, which resulted in accuracy rates of $98 \%$ to $100 \%$. Table 3 shows the results are far superior to other available measurement technologies; users of the movement flow measurement system are generally very satisfied with an accuracy of more than $90 \%$, which indicates that the proposed laser measurement system is valuable, also as a statistic figure. Such high outcome is possible because the laser sensors tracked the passengers fifteen times every second and sent the data. Furthermore, even when the passengers were overlapped from one perspective, the six sensors measured them from different perspectives, whose data were processed through a software logic that distinguished individual targets.

Table 3. Accuracy of the Count

\begin{tabular}{|c|c|c|c|c|c|c|c|c|c|}
\hline No & $\begin{array}{c}\text { Arrival } \\
\text { Date }\end{array}$ & $\begin{array}{c}\text { Arrival } \\
\text { Gate }\end{array}$ & $\begin{array}{l}\text { Flight } \\
\text { Code }\end{array}$ & Departure & $\begin{array}{c}\text { Arrival } \\
\text { Time }\end{array}$ & $\begin{array}{r}\text { No. of } \\
\text { (Pa: } \\
\text { Crew }\end{array}$ & $\begin{array}{l}\text { sengers } \\
\text { ger }+ \\
\text { ransit) }\end{array}$ & Count & $\begin{array}{c}\text { Accurac } \\
\mathbf{y}(\%)\end{array}$ \\
\hline 1 & $4 / 26$ & A & BX392 & Hong Kong & $6: 24$ & 114 & \multirow{5}{*}{963} & \multirow{5}{*}{950} & \multirow{5}{*}{$99 \%$} \\
\hline 2 & $4 / 26$ & A & BX382 & Macao & $6: 14$ & 71 & & & \\
\hline 3 & $4 / 26$ & A & KE618 & Hong Kong & $6: 04$ & 136 & & & \\
\hline 4 & $4 / 26$ & A & BX712 & Cebu & $6: 26$ & 186 & & & \\
\hline 5 & $4 / 26$ & A & VN422 & $\begin{array}{l}\text { Ho Chi } \\
\text { Minh }\end{array}$ & $6: 43$ & 284 & & & \\
\hline No & $\begin{array}{c}\text { Arrival } \\
\text { Date }\end{array}$ & $\begin{array}{c}\text { Arrival } \\
\text { Gate }\end{array}$ & $\begin{array}{l}\text { Flight } \\
\text { Code }\end{array}$ & Departure & $\begin{array}{c}\text { Arrival } \\
\text { Time }\end{array}$ & \multicolumn{2}{|c|}{$\begin{array}{c}\text { No. of Passengers } \\
\text { (Passenger + } \\
\text { Crew + Transit) }\end{array}$} & Count & $\begin{array}{c}\text { Accurac } \\
\text { y (\%) }\end{array}$ \\
\hline 1 & $4 / 27$ & A & BX342 & Xi'an & $06: 35$ & 152 & \multirow{12}{*}{1,687} & \multirow{12}{*}{1,683} & \multirow{12}{*}{$98 \%$} \\
\hline 2 & $4 / 27$ & A & BX392 & Hong Kong & 06:05 & 165 & & & \\
\hline 3 & $4 / 27$ & A & BX382 & Macao & 06:08 & 90 & & & \\
\hline 4 & $4 / 27$ & A & KE618 & Hong Kong & 06:11 & 129 & & & \\
\hline 5 & $4 / 27$ & A & BX798 & Taipei & $06: 14$ & 106 & & & \\
\hline 6 & $4 / 27$ & A & BX712 & Cebu & $06: 32$ & 182 & & & \\
\hline 7 & $4 / 27$ & A & QV915 & Vientiane & $06: 52$ & 100 & & & \\
\hline 8 & $4 / 27$ & A & BX722 & Siem Reap & $06: 49$ & 165 & & & \\
\hline 9 & $4 / 27$ & A & TG650 & Bangkok & 07:00 & 242 & & & \\
\hline 10 & $4 / 27$ & A & BX732 & Da Nang & $06: 56$ & 178 & & & \\
\hline 11 & $4 / 27$ & A & $\underset{)}{\mathrm{BX} 714^{* 1}}$ & Cebu & 07:10 & 56 & & & \\
\hline 12 & $4 / 27$ & A & $\underset{)}{O Z 706^{* 1}}$ & Manila & $07: 32$ & 122 & & & \\
\hline No & $\begin{array}{c}\text { Arrival } \\
\text { Date }\end{array}$ & $\begin{array}{c}\text { Arrival } \\
\text { Gate }\end{array}$ & $\begin{array}{l}\text { Flight } \\
\text { Code }\end{array}$ & Departure & $\begin{array}{c}\text { Arrival } \\
\text { Time }\end{array}$ & \multicolumn{2}{|c|}{$\begin{array}{c}\text { No. of Passengers } \\
\text { (Passenger + } \\
\text { Crew + Transit) }\end{array}$} & Count & $\begin{array}{c}\text { Accurac } \\
\mathbf{y}(\%)\end{array}$ \\
\hline 1 & $4 / 28$ & A & BX392 & Hong Kong & 06:04 & 156 & \multirow{2}{*}{1,809} & \multirow{2}{*}{1,814} & \multirow{2}{*}{$99 \%$} \\
\hline 2 & $4 / 28$ & A & BX382 & Macao & 06:01 & 78 & & & \\
\hline
\end{tabular}




\begin{tabular}{|c|c|c|c|c|c|c|c|}
\hline 3 & $4 / 28$ & A & KE618 & Hong Kong & $06: 06$ & 134 \\
\hline 4 & $4 / 28$ & A & BX798 & Taipei & $06: 20$ & 154 \\
\hline 5 & $4 / 28$ & A & BX712 & Cebu & $06: 43$ & 197 \\
\hline 6 & $4 / 28$ & A & VN422 & $\begin{array}{c}\text { Ho Chi } \\
\text { Minh }\end{array}$ & $06: 32$ & 285 \\
\hline 7 & $4 / 28$ & A & VJ982 & Hanoi & $06: 39$ & 180 \\
\hline 8 & $4 / 28$ & A & BX722 & Siem Reap & $06: 46$ & 181 \\
\hline 9 & $4 / 28$ & A & BX374 & Sanya & $07: 13$ & 156 \\
\hline 10 & $4 / 28$ & A & BX611 & Guam & $07: 20$ & 146 & \\
\\
\hline 11 & $4 / 28$ & A & BX714 & Cebu & $07: 16$ & 142 & \\
\end{tabular}

\subsection{Results of Stay Time Measurement}

Based on the four days of measurement data, Figure 6 shows the current study analyzed the trends in the stay time near carousel \#1. In order to identify the entire process from the flight arrival to passengers exiting with their baggage, the data was analyzed and visualized.

The analysis results allow the users to accurately predict and identify the two things that were unclear in the past.

\section{(1) Identification of Congestion near the Carousel \#1}

The area occupation rate reached 50\% near the carousel \#1 at 7:37 on April 28, when the number of passengers was the highest. The heat map shows that many people are gathered near the right-hand side gate and on the path to the exit. This indicates that these sections are especially congested, posing dangers of the passengers running into each other; the airport needs a plan to relieve congestion and improve passenger safety.

Carousel \#1 is an old model, with the heat map indicating that the setup of the machine is not attracting many passengers to the inner waiting area. That is, the solution can provide comparisons in the future when new models of carousel \#1 are introduced.

\section{(2) Identification of Stay Time for Baggage Claim}

The area occupation rate graph at 7:00 on April 26 shows that it took maximum forty minutes for passengers to claim and go into the arrival area during the early morning hours of the day. Some passengers claimed the baggage as quickly as in ten minutes.

These numbers may be related to the number of passengers on each flight; shortening the time required to claim bags can be used as a key performance indicator (KPI) item, in order to improve the customer comfort and satisfaction. After the movement flow measurement results are converted to a KPI item, in conjunction with the number of passengers on flight, number of baggage, and the length of carousel \#1 operation time, the data can be used to identify movement flow improvement solutions reduce wait times and congestion levels, thereby quantifying enhancements in the customer service quality.

The interpreted movement flow data can also be processed with information from airline such as flight arrival time, arrival gate, and baggage processing start time to identify how which airlines are performing in relation to passenger flow. 


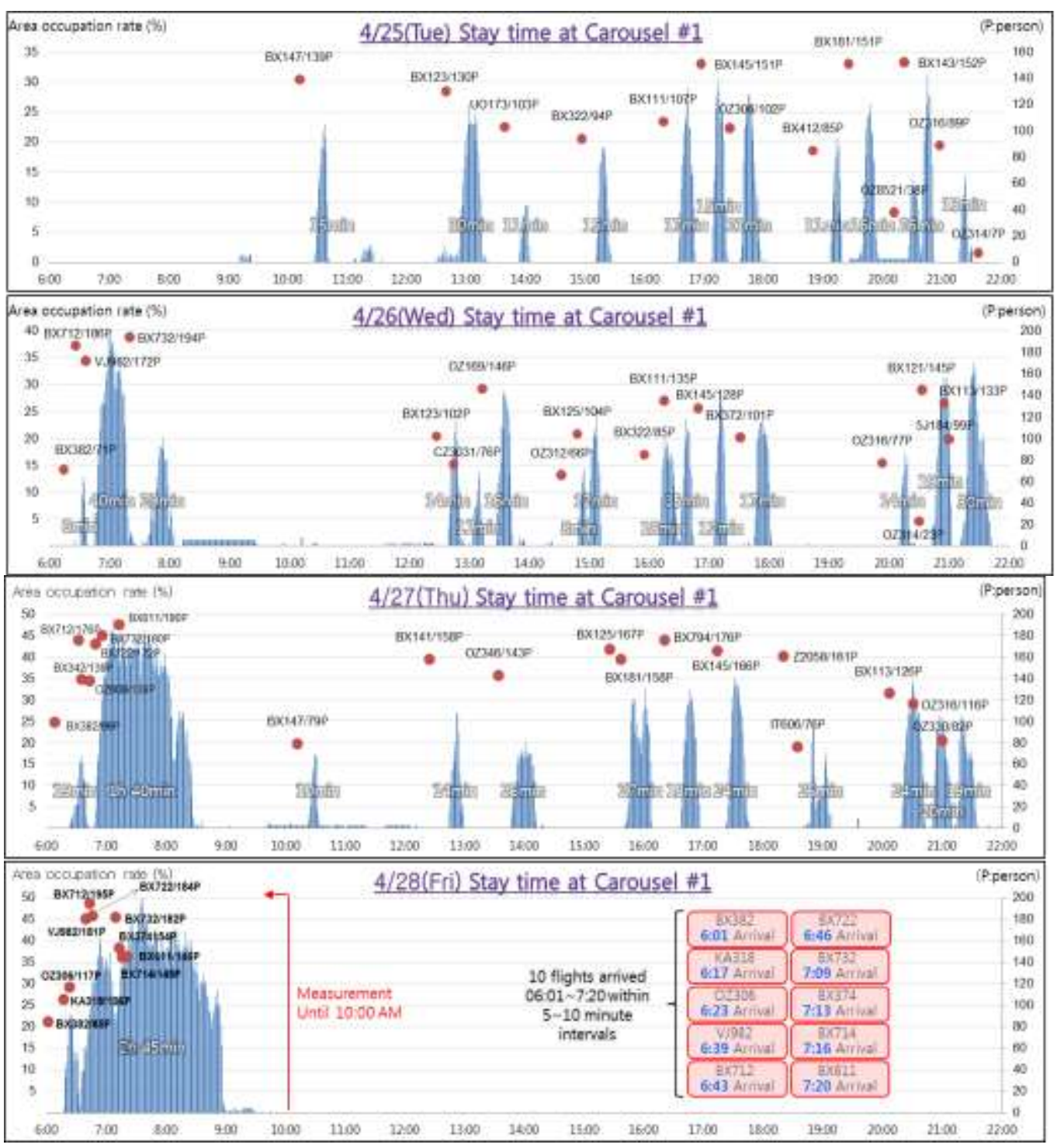

Figure 6. Results of Stay Time Measurement

\subsection{Movement Flow and Trajectory}

Using a technology in which the movements of the targets are tracked to visualize their trajectory until they exit the laser sensor range, the congestion levels can be identified as shown in Figure 7. This method also provides comprehensive information regarding which areas people move. Figure 7 visualizes the trajectory of passengers from passing through the entrance gate until they claim their baggage. Each pink dot refers to a passenger, and the blue lines are the trajectory of the passenger that is, their line of movement. The visualized information indicates that during the busiest period, passengers are crowded at the outer edge of the carouse \#l, rather than deeper in the left- and righthand sides. This information shows that the congestion in the pathway from the righthand side entrance gate to the exit gate needs to be resolved in order to prevent passengers from running into each other. 


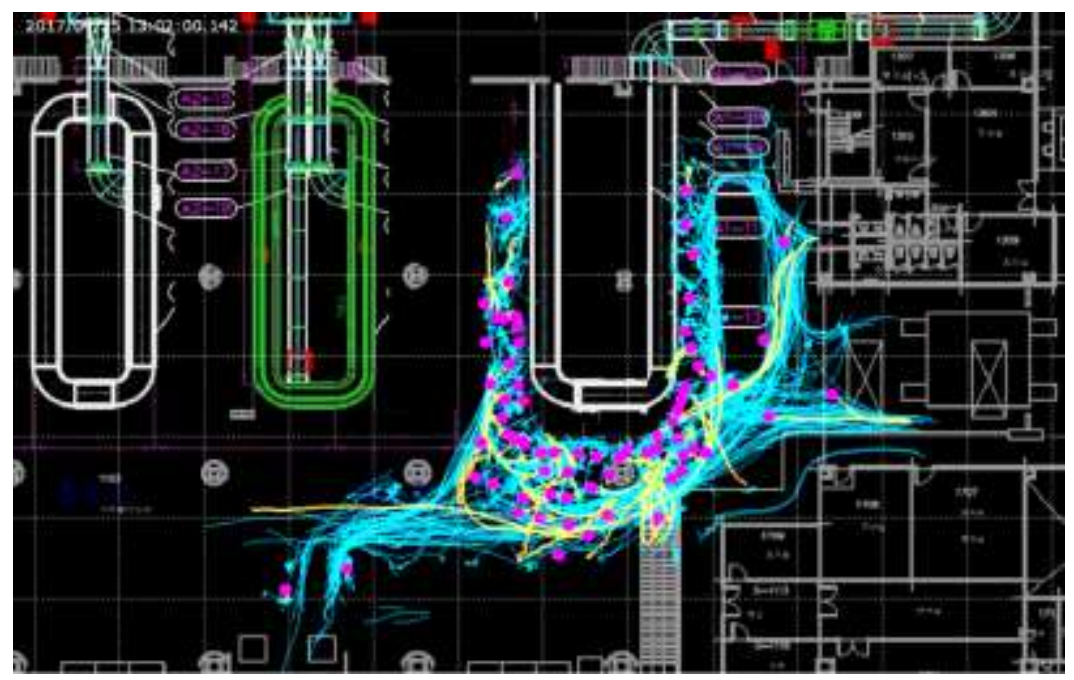

Figure 7. Visualization of the Movement Trajectories

\subsection{Heat Map}

The heat map is a visualization technique of color-coding individual indices in order to analyze the relationship between the movement flow of people and other factors. Figure 8 On the heat map, colors closer to blue indicate that the stay time of passengers in the grid is closer to zero seconds within one minute, while those closer to red indicates stay times closer to sixty seconds within the one minute. In other words, redder colors indicate more congested sections and bluer colors show relatively less congested spaces.

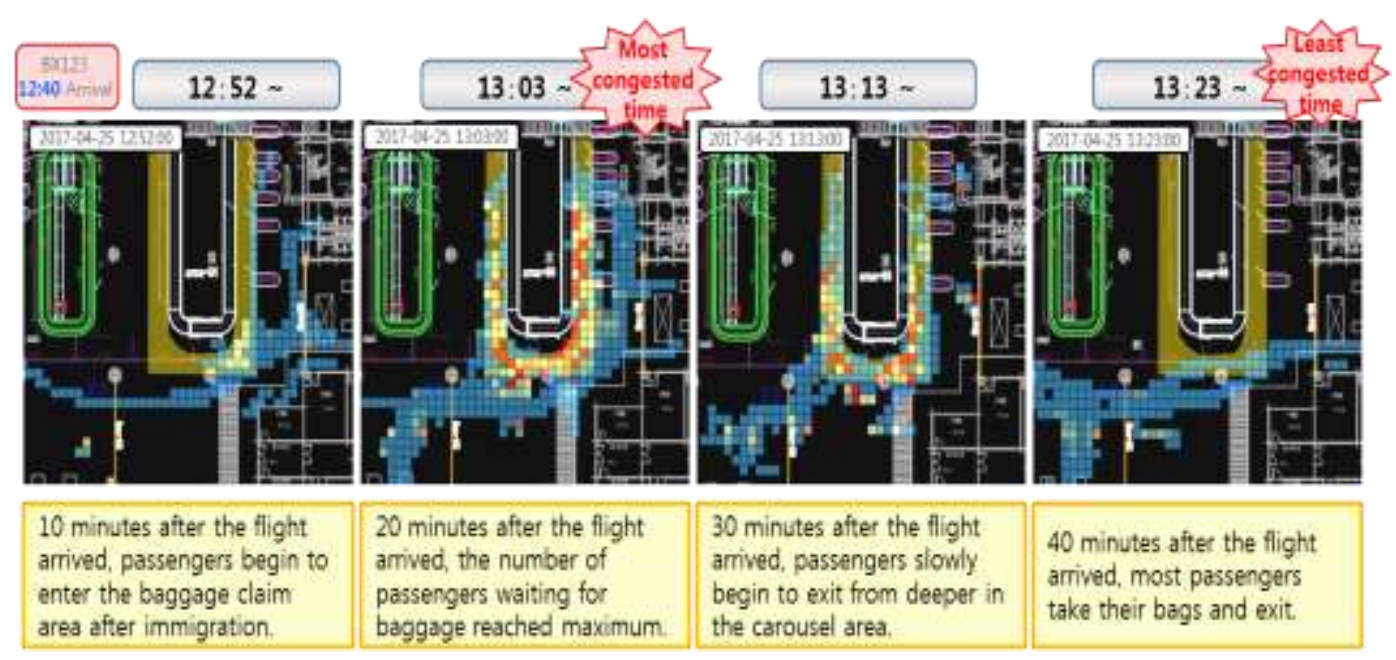

Figure 8. Visualization of Heat Map

\section{Conclusion}

With the development of IoT technologies, recent trends in research show more studies are being conducted to measure location information of people, analyze their movement flow and congestion levels in certain spaces, and then use the resulting information to improve workflow and customer service. Previous research efforts on movement flow measurement, however, have been for purely academic purposes or verification of theories, and their results were not adequate for applying to actual businesses. The wireless LAN measurement method which was initially thought to be most convenient yielded the insufficient amount of collected data because of the targets had to own a smartphone and turn on its Bluetooth feature. Using CCTV was also problematic because 
it violated the targets' privacy and laws related to the subject, as the method exposed people's faces. Lastly, the RFID technology had insufficient detection ranges, raising the cost of devices for measuring wide areas. The current study originated in an attempt to resolve the inadequacies posed by conventional detection methods; the researchers developed a movement flow measurement system using laser sensors, devices that do not violate privacy regulations and collect data conveniently and accurately without requiring the targets to carry a special device. The system was set up in an airport, where the experimental measurement was conducted for four days. The results show that the issues regarding measurement accuracy and privacy were resolved by using the proposed system [19].

First, the system counted the number of passengers with more than $98 \%$ accuracy, which is far superior to other counting systems. It is also able to calculate stay times and congestion levels in $1 \mathrm{~m} \times 1 \mathrm{~m}$ sections in the grid. The proposed system is also capable of not only tracking the directions of movement trajectories, but also the speed of the targets. Synchronizing the collected measurement data and the flight arrival time information also made it possible to comprehensively visualize into a graph the time it requires for a passenger to land on a flight, claim the baggage, and exit into the arrival hall.

Despite the high volume of data, with fifteen data points transferred from the sensors to the PC, which were then sent to the server to process and analyze, the system operated in a stable manner. The countless data points, in turn, reflect the high accuracy of the accuracy in the database.

Second, the system eliminated privacy concerns as it only collects $\mathrm{X}$ - and $\mathrm{Y}$-axis location coordinates, time, speed, and vector values, which are attached to randomly generated trajectory IDs. As the measure is minimally intrusive, the passengers were not aware that their movement was being measured, and the study confirmed that there is zero potential for a leak in personal information, as the system does not collect any personal information from individual passengers.

Using the method proposed in the current study will enable companies and organizations to analyze their customers' movement flow in public spaces (airport, metro, commercial facilities, etc.,) without violating privacy while covering wide areas with low cost, aided by convenient installation and operation methods. Conventional movement flow measurement system required much time and manual effort in data collection, with analysis and interpretation conducted using experiential knowledge. The system proposed in the current study, on the other hand, enables the user to identify problems with movement flow in a space and find areas for improvement with minimal effort, making it a useful tool for enhancing customer service. The current study used six 2D laser sensors to track and measure the movement flow of passengers in an airport. The experiment resulted in the accuracy higher than $98 \%$ and proved that the proposed system can measure congestion and target stay time in designated areas, in addition to expressing the data with various visualization techniques. In terms of trajectory, however, it was difficult to completely track targets from endpoint-to-endpoint, due to obstacles such as pillars.

The current research may serve as a guideline for establishing a new and valuable technique for accurately tracking and analyzing the movement flow of people in airports, train stations, and department stores. The future research direction is to employ 3D laser sensors in movement flow measurement, in order to obtain even higher accuracy than that from this study, which used 2D laser sensors.

\section{References}

[1] M.-b. Kim, "A Study on the Improvement of Algorithm in Consumer Moving Path Similarity Measurement Using Beacon Data", Soongsil University Ph.D. dissertation, (2017).

[2] S.-il Kim and H.-s. Kim, "A Study on Indoor Positioning System Based RFID for LBS", Korean Institute of Information Scientists and Engineers 2011 Korea Computer Science Academic Conference Journal, vol. 38, no. (1(D)), (2011). 
[3] N. Hyeon "Measuring signal analysis and comparison system using Bluetooth LE Beacon", Hannam University Master's degree dissertation, (2015).

[4] N. Angela Song Ie and W.-j. Lee. "Accuracy evaluation of ZigBee's indoor localization algorithm", Korean Society for Internet Information Journal, vol. 11, no. 1, (2009).

[5] Y.-s. Bok, "Accurate motion estimation and mapping based on sensor fusion of camera and laser scanner", Korea Advanced Institute of Science and Technology Master's degree dissertation, (2005).

[6] A. Jae-myeong, "A study on real-time preference goods recommendation using RFID-based shopping moving line analysis", Soongsil University Ph.D. dissertation, (2005).

[7] Y. Jae-jun, "Trends in Technical Development and Standardization of Indoor Location Based Services", TTA Journal, vol. 157, (2015).

[8] K.-y. Lee, “A Study on Accuracy Enhancement of Indoor Local Positioning System Based on Zigbee", Journal of Korean Institute of Information Technology, vol. 8, no. 5, (2010).

[9] I. Sang-min, "Design of Intelligent Preference Goods Recommendation System Using Customer's Shopping Location Tracking and Moving Line Analysis on based RFID", Chungwoon University Master's degree dissertation, (2008).

[10] I. Il-gwon, "Indoor positioning system using BLE beacon and extended Kalman filter", Hannam University Ph.D. dissertation, (2015).

[11] V. L. Babu, R. Batta and L. Lin, "Passenger grouping under constant threat probability in an airport security system", European Journal of Operational Research, no. 168, (2006), pp. 633-644.

[12] L. Brunetta and G. Romanin-Jacur, "Passenger and baggage flow in an airport terminal: a flexible simulation model", Journal of Air Traffic Management, no. 6, (1999), pp. 361-363.

[13] D. Curcio, F. Longo, G. Mirabelli and E. Pappoff, "Passengers' Flow Analysis and Security Issues in Airport Terminals Using Modeling \& Simulation”, University of Calabria 87036, Rende (CS), Italy, (2007).

[14] F. Tsunoda, "Customer-Oriented Evaluation Method of Railway Performance", 6th International Conference on Railway Operations Modelling and Analysis, RailTokyo2015, (2015).

[15] M. R. Gatersleben and S. W. van der Weij, "Analysis and simulation of passenger flows in an airport terminal", In Proceedings of the 1999 Winter Simulation Conference (Squaw Peak, AZ, Dec.5-8). Phoenix, AZ, (1999), pp. 1226-1231.

[16] M. Fujiwara, "Passenger flow simulator for systematic optimization of station layout and train timetable", $6^{\text {th }}$ International Conference on Railway Operations Modelling and Analysis, RailTokyo2015, (2015).

[17] N. Ki-in, B. Jae-min, R. Myong-chan and S. Beom-su, "Fusion of multiple 2D LiDAR and RADAR for object detection and tracking in all directions", International Conference on Connected Vehicles and Expo (ICCVE), (2014), pp. 1058-1059.

[18] E. S. Souza, "Tracking System for Urban Buses with People Flow Management", IEEE Latin America Transactions, vol. 9, no. 6, (2011), pp. 944-949.

[19] S. H. Lee, H. T. Lee, P. Nhu Quynh and G. Y. Gim, "A Study on the Analysis and Visualization Method of Passenger Flow Using Laser Sensor", Advanced Science and Technology Letters ASTL 150, Ho Chi Minh, Vietnam, (2018) February 1-3.

\section{Authors}

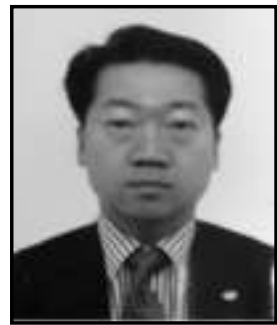

Seok-Hee Lee, (chris@1ghitachi.co.kr)

$\mathrm{He}$ works as a Managing Director at the Department of Solution \& Service Business Divisionat LG Hitachi Ltd, and he is studying in Soongsil University Graduate School of IT Policy Management. His research areas are IoT, Big Data, Artifical Inteligence, Fintech and Payment Solution with Biometrics, etc.

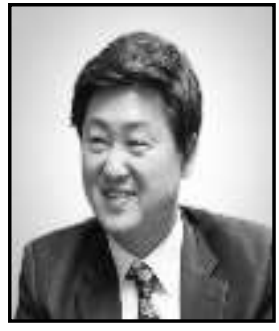

Hyung-Taek Lee, (htlee@innotium.com)

He works as a CEO at Innotium Inc, Development Company for Ransomawre Defense and Data Security and he works for Ransomeware Infringement Response Center (RanCERT), and he is studying in Soongsil University Graduate School of IT Policy Management. His research areas are Cyber Security, Data Security, Cloud Plaform, Big Data, Artifical Inteligence, and Software Engineering. 


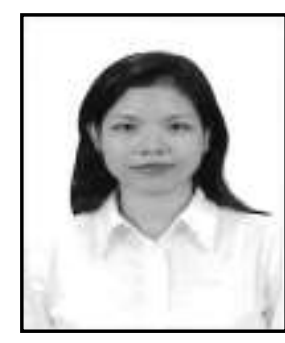

Nhu-Quynh Phan, (phannhuquynh@hotmail.com)

She got Master's degree in Business Administration at Soongsil University. She is currently studying for a $\mathrm{PhD}$ at Department of Business Administration of Soongsil University. Her interest research areas are IoT, Collective Intelligence.

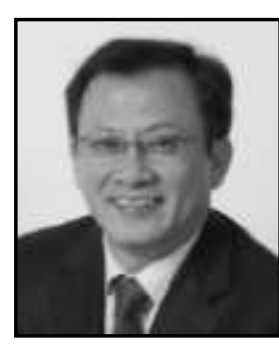

Gwang-Yong Gim, (gygim@ssu.ac.kr)

$\mathrm{He}$ works as a professor at the Department of Business Administration of Soongsil University. Dr. Gim has been interested in research such as $4^{\text {th }}$ Industry Revolution, ICT ODA, intellectual property rights, service science, big data analysis, $\mathrm{S} / \mathrm{W}$ industrial policy, and open innovation. He published a number papers on journals such as Information Science, Fuzzy sets and System, journals of society of management information systems, and journals of management science. 
International Journal of Control and Automation

Vol. 11, No. 5 (2018) 